Supporting Information for

\title{
Compatibilization of All-Conjugated Polymer Blends for Organic Photovoltaics
}

\author{
Florian Lombeck ${ }^{1,2}$, Alessandro Sepe ${ }^{3}$, Ralf Thomann ${ }^{4}$, Richard H. Friend ${ }^{1}$, Michael \\ Sommer ${ }^{2,4,5^{*}}$
}

${ }^{1}$ Cavendish Laboratory, Department of Physics, University of Cambridge, 19 JJ Thomson Avenue, Cambridge CB3 0HE, UK.

${ }^{2}$ Makromolekulare Chemie, Universität Freiburg, Stefan-Meier-Straße 31, 79104 Freiburg, Germany.

${ }^{3}$ Adolphe Merkle Institute, Chemin des Verdiers 4, CH-1700, Fribourg, Switzerland.

${ }^{4}$ Freiburger Materialforschungszentrum, Stefan-Meier-Straße 21, 79104 Freiburg, Germany.

${ }^{5}$ FIT, Freiburger Zentrum für interaktive Werkstoffe und bioinspirierte Technologien, GeorgesKöhler-Allee 105, 79110 Freiburg, Germany. 


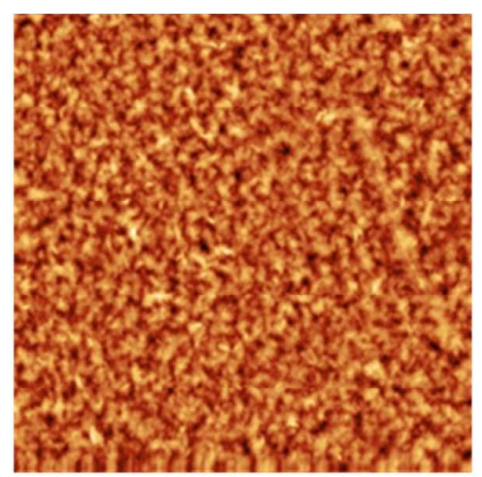

$0 \%$

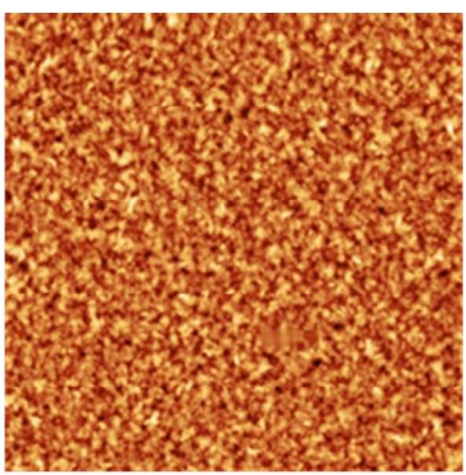

$2 \%$

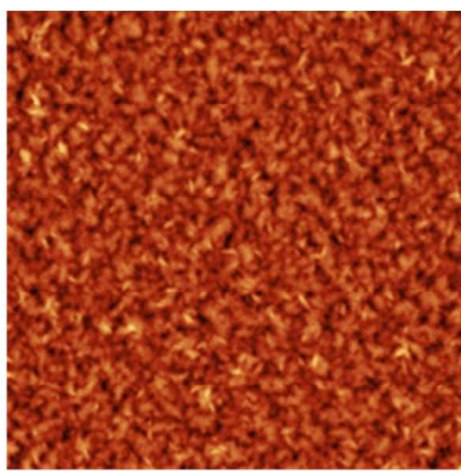

$5 \%$ SF-BCP

Figure SI-1. AFM images of binary and ternary blends (SF-PCDTBT:P3HT 1:1 + X\% SFBCP-20) before thermal treatment. The rms roughness obtained was identical for all film surfaces. RMS roughness for all films is $5.6 \pm 0.5 \mathrm{~nm}$.

The static contact angle of water and n-octane on annealed thin films of binary polymer blends, SF-PCDTBT:P3HT, with variation in donor-acceptor ratio were measured. This measurement technique is sensitive towards the composition and structure of the film-air interface and revealed a preferential formation of a SF-PCDTBT capping layer at the air-film interface.

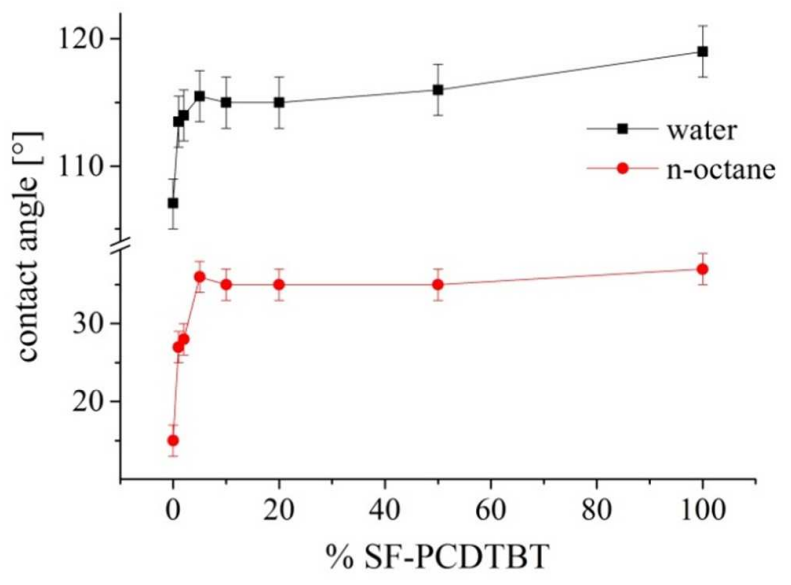

Figure SI-2. Contact angle of a water (black) droplet $/ n$-octane (red) droplet on the polymer film as a function of wt.- $\%$ SF-PCDTBT in the binary blend; film thickness is $65-70 \mathrm{~nm}$. 
This circumstance is supported by the observed formation of a bilayer structure in unannealed SF-PCDTBT:P3HT (1:1) blend films. UV-vis spectra of films treated with $1,2,4-$ Trifluorobenzene (TFB) revealed a selective solubility of SF-PCDTBT in TFB as can be followed by UV-vis absorption where only minor contributions of SF-PCDTBT can be detected below $400 \mathrm{~nm}$. Additionally, the unannealed film exhibits no macrophase separated features in the AFM image and an average film thickness of $80 \mathrm{~nm}$, the film thickness is reduced to $40 \mathrm{~nm}$ after solvent treatment whereas the film surface topography remains featureless. 
a)

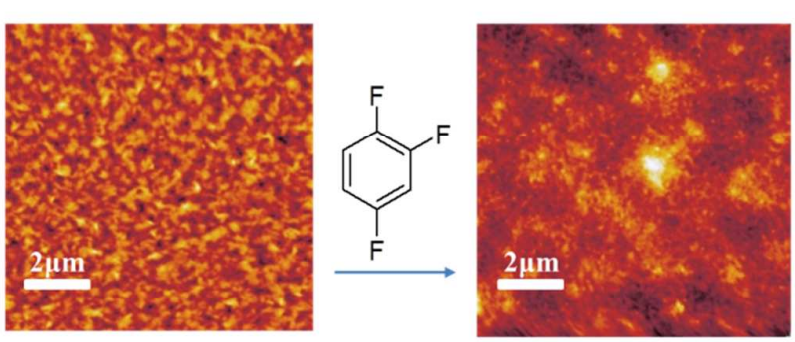

b)

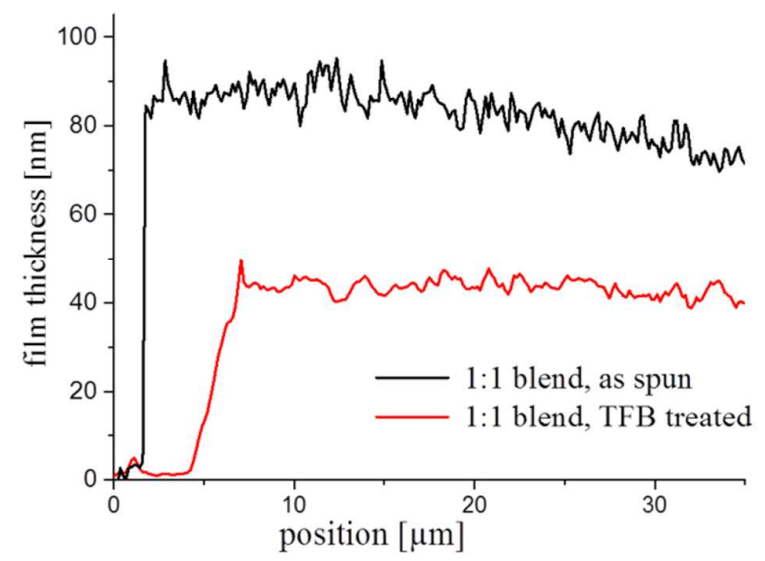

c)

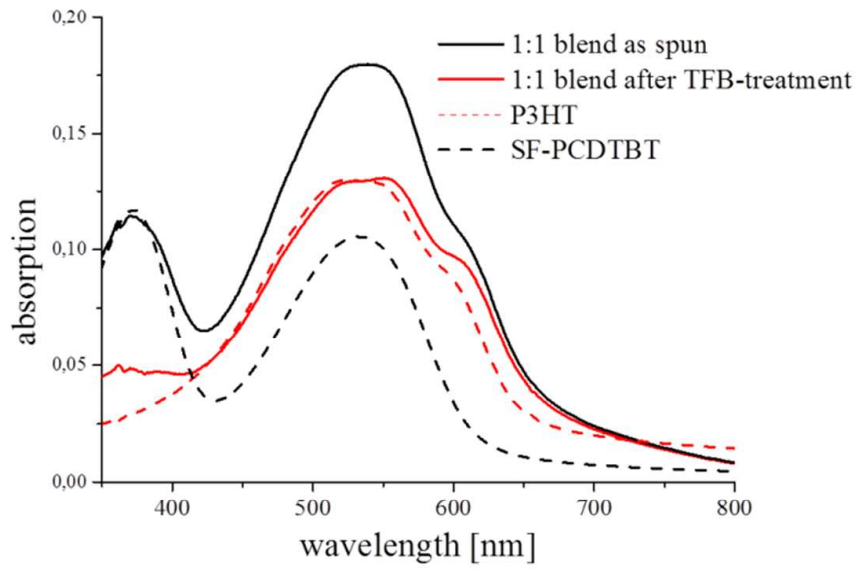

d)

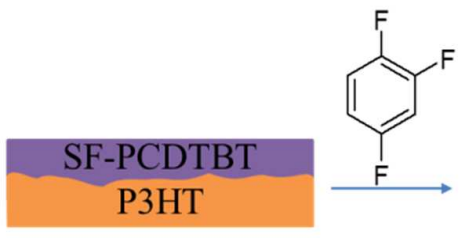

Figure SI-3. Blend of P3HT:SF-PCDTBT (1:1 by weight) a) AFM height image of the blend;

b) height profile of the blend film, as spun (black) and TFB treated (red); c) absorption spectra of the binary blend, as spun (solid black) and TFB treated (solid red) as well as the individual spectra of P3HT (dashed red) and SF_PCDTBT (dashed black); d) a schematic illustration of the observations. 


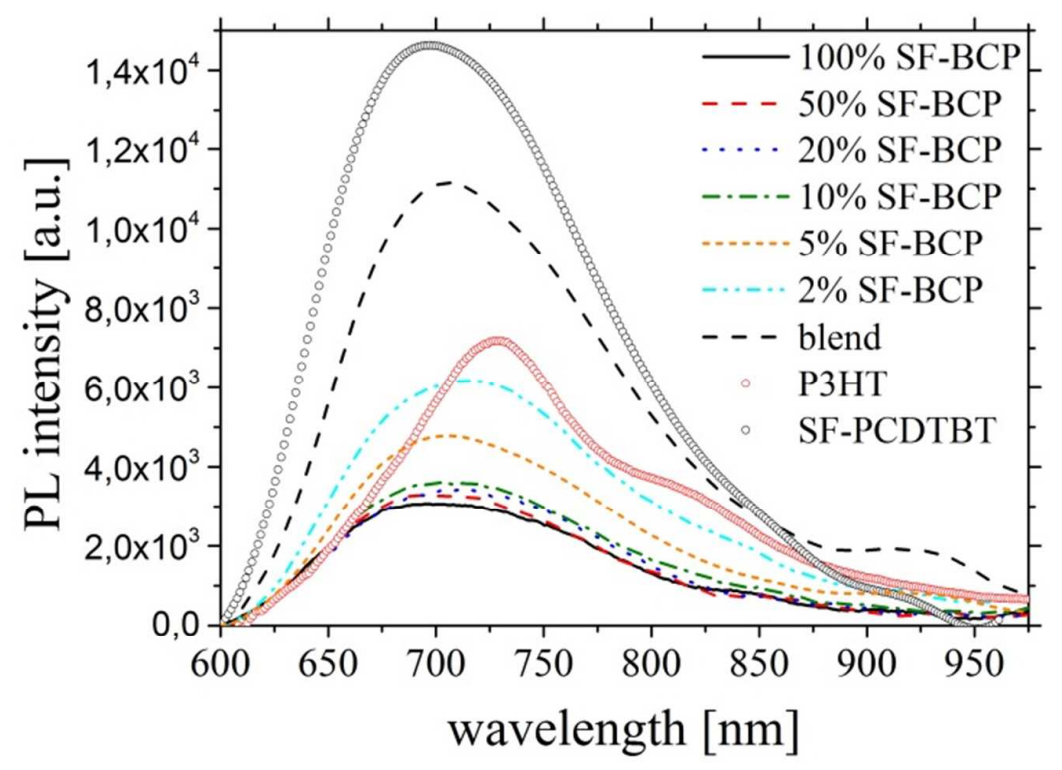

Figure SI-4. Photoluminescence spectra of the binary blend compatibilized with SF-BCP-20.

a)

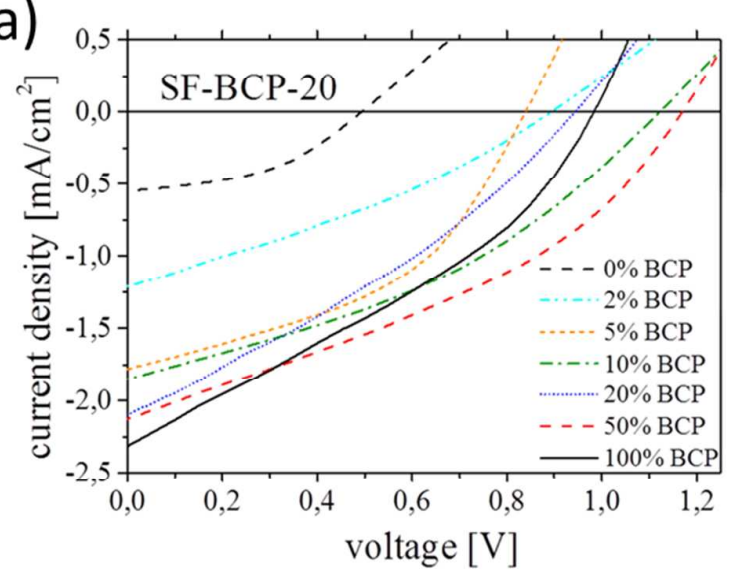

b)

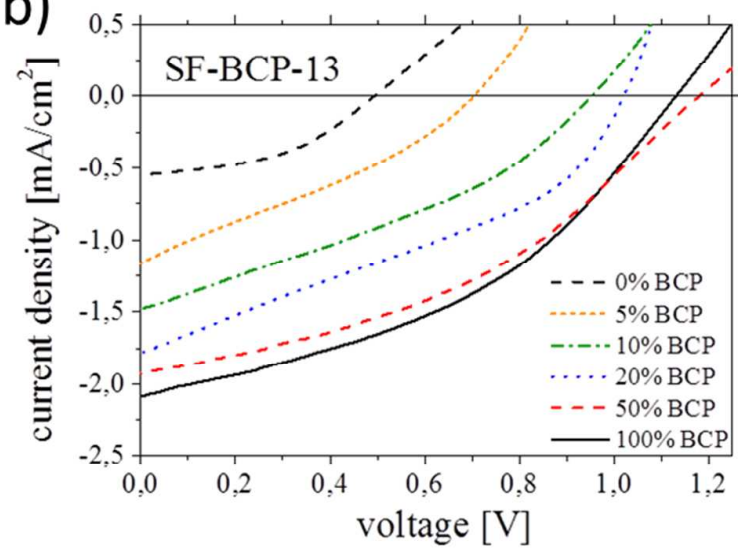

Figure SI-5. $J-V$ curves of OPV devices using compatibilized blends as active layer; a) compatibilization with SF-BCP-20, b) compatibilization with SF-BCP-13 
a)

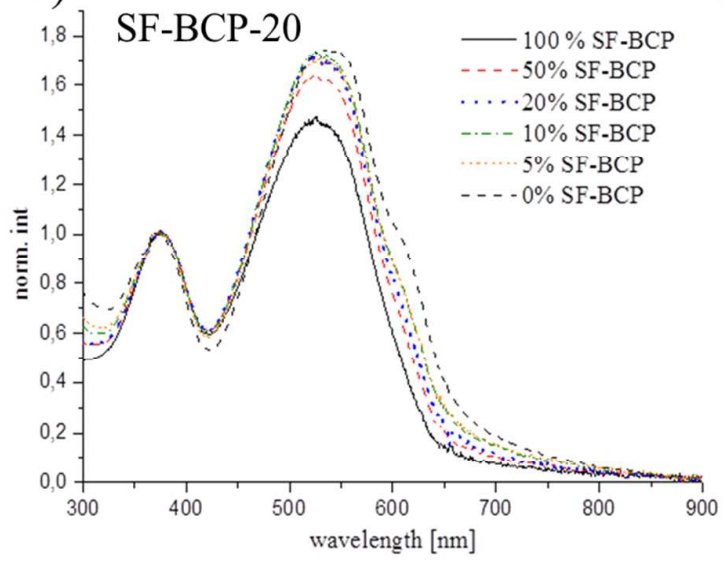

b)

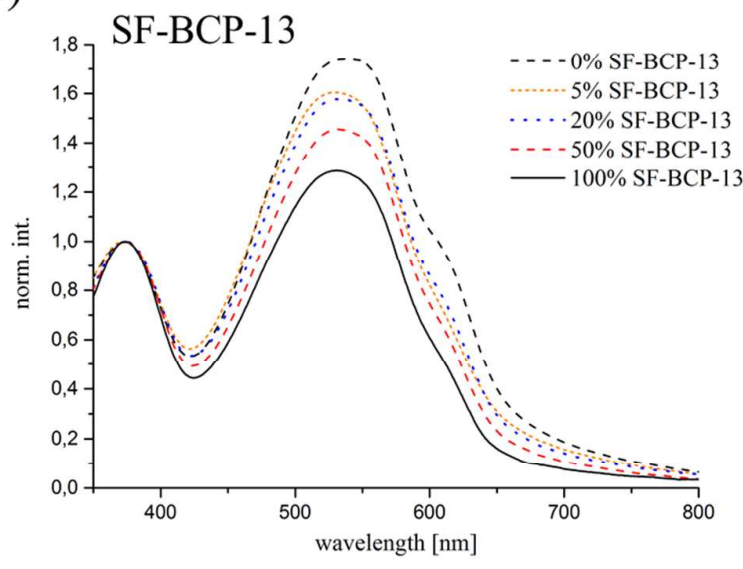

Figure SI-6. Absorption spectra of the compatibilized blends; a) compatibilization with SFBCP-20; b) compatibilization with SF-BCP-13.

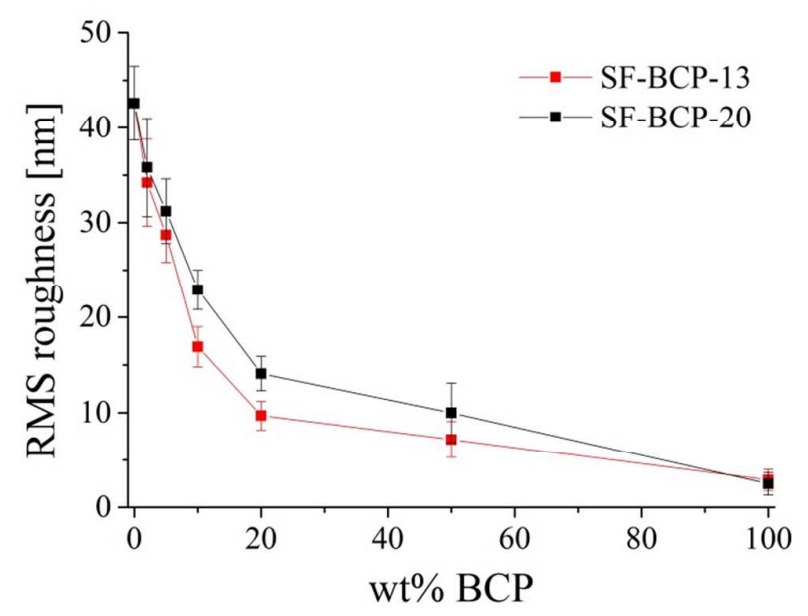

Figure SI-7. Root-mean-square roughnesses of compatibilized thin films as a function of wt.- $\%$ BCP after thermal annealing at $260^{\circ} \mathrm{C}$. 
Table SI-1. Energy level values of the discussed (co-)polymers.* determined by UPS, \# determined by absorption onset and cyclic voltammetry, ${ }^{\S}$ determined by GPC measurements.

\begin{tabular}{ccccccc}
\hline Polymer & $\begin{array}{c}\text { HOMO } \\
{[\mathrm{eV}]^{*}}\end{array}$ & $\begin{array}{c}\text { LUMO } \\
{[\mathrm{eV}]^{* *}}\end{array}$ & $\begin{array}{c}\mathrm{Eg} \\
{[\mathrm{eV}]^{\#}}\end{array}$ & $\begin{array}{c}M_{n} \\
{[\mathrm{~kg} / \mathrm{mol}]} \\
\S\end{array}$ & $\begin{array}{c}M_{w} \\
{[\mathrm{~kg} / \mathrm{mol}]} \\
\S\end{array}$ & $\mathrm{Ð}^{\S}$ \\
\hline P3HT & -4.7 & -2.8 & $1.90 \pm 0.03$ & 19.1 & 22.5 & 1.18 \\
H-PCDTBT & -5.35 & -3.7 & $1.88 \pm 0.02$ & 22.0 & 68.6 & 3.12 \\
hex-PCDTBT & -5.5 & -3.5 & $2.01 \pm 0.02$ & 46.9 & 100.4 & 2.14 \\
SF-PCDTBT & -5.8 & -3.8 & $2.01 \pm 0.02$ & 37.1 & 58.9 & 1.59 \\
\hline
\end{tabular}

Table SI-2. Photoluminescence Quantum Efficiencies (PLQE) of homo- and copolymers and the corresponding 1:1 blends; PLQEs were measured from films with thicknesses of 65-70nm.

\begin{tabular}{lcc}
\hline & Copolymer & $\begin{array}{c}\text { Blend with P3HT } \\
(1: 1)\end{array}$ \\
& & \\
\hline H-PCDTBT & $8.3 \pm 1.5$ & $3.8 \pm 1.5$ \\
hex-PCDTBT & $18.4 \pm 1.5$ & $3.2 \pm 1.5$ \\
SF-PCDTBT & $11.8 \pm 1.5$ & $8.8 \pm 1.5$ \\
P3HT & $3.9 \pm 1.5$ & --- \\
\hline
\end{tabular}


Table SI-3. Photovoltaic device characteristics and PLQE values for the ternary blends compatibilized with SF-BCP-13. Jsc: short circuit current density; Voc: open circuit voltage; FF: fill factor; PCE: power conversion efficiency; PLQE: photoluminescence quantum efficiency.

\begin{tabular}{cccccc}
\hline SF-BCP-13 & $\begin{array}{c}V_{O C} \\
{[\mathrm{~V}]}\end{array}$ & $\begin{array}{c}J_{S C} \\
{\left[\mathrm{~mA} / \mathrm{cm}^{2}\right]}\end{array}$ & $\begin{array}{c}\text { FF } \\
{[\%]}\end{array}$ & $\begin{array}{c}\text { PCE } \\
{[\%]}\end{array}$ & $\begin{array}{c}\text { PLQE } \\
{[\%]}\end{array}$ \\
\hline $100 \%$ SF-BCP-13 & $1.09 \pm 0.05$ & $2.06 \pm 0.14$ & $39.2 \pm 2.0$ & $0.91 \pm 0.06(0.97)$ & $3.3 \pm 1.5$ \\
$50 \%$ SF-BCP-13 & $1.12 \pm 0.08$ & $1.87 \pm 0.16$ & $37.0 \pm 1.6$ & $0.77 \pm 0.11(0.88)$ & $4.9 \pm 1.5$ \\
$20 \%$ SF-BCP-13 & $0.97 \pm 0.10$ & $1.78 \pm 0.18$ & $36.7 \pm 1.2$ & $0.63 \pm 0.12(0.70)$ & $3.9 \pm 1.5$ \\
$10 \%$ SF-BCP-13 & $0.90 \pm 0.08$ & $1.48 \pm 0.17$ & $35.3 \pm 1.9$ & $0.47 \pm 0.10(0.52)$ & $4.6 \pm 1.5$ \\
$5 \%$ SF-BCP-13 & $0.78 \pm 0.05$ & $1.12 \pm 0.21$ & $34.1 \pm 2.7$ & $0.30 \pm 0.08(0.34)$ & $5.9 \pm 1.5$ \\
$0 \%$ SF-BCP-13 & $0.48 \pm 0.08$ & $0.55 \pm 0.05$ & $32.7 \pm 3.8$ & $0.09 \pm 0.05(0.12)$ & $8.8 \pm 1.5$ \\
\hline
\end{tabular}

Table SI-4. Photovoltaic device characteristics and PLQE values for the ternary blends compatibilized with SF-BCP-20. JSc: short circuit current density; Voc: open circuit voltage; FF: fill factor; PCE: power conversion efficiency; PLQE: photoluminescence quantum efficiency.

\begin{tabular}{cccccc}
\hline SF-BCP-20 & $\begin{array}{c}\text { Voc } \\
{[\mathrm{V}]}\end{array}$ & $\begin{array}{c}J_{S C} \\
{\left[\mathrm{~mA} / \mathrm{cm}^{2}\right]}\end{array}$ & $\begin{array}{c}\text { FF } \\
{[\%]}\end{array}$ & $\begin{array}{c}\text { PCE } \\
{[\%]}\end{array}$ & $\begin{array}{c}\text { PLQE } \\
{[\%]}\end{array}$ \\
\hline $100 \%$ SF-BCP-20 & $0.96 \pm 0.13$ & $-2.21 \pm 0.15$ & $34.6 \pm 4.5$ & $0.74 \pm 0.15$ & $3.8 \pm 1.5$ \\
$50 \%$ SF-BCP-20 & $1.08 \pm 0.9$ & $-2.07 \pm 0.14$ & $36.2 \pm 4.4$ & $0.89 \pm 0.18$ & $2.6 \pm 1.5$ \\
20\% SF-BCP-20 & $0.93 \pm 0.13$ & $-2.10 \pm 0.14$ & $36.0 \pm 4.7$ & $0.71 \pm 0.14$ & $3.2 \pm 1.5$ \\
$10 \%$ SF-BCP-20 & $1.01 \pm 0.14$ & $-1.85 \pm 0.12$ & $36.8 \pm 5.1$ & $0.75 \pm 0.12$ & $3.4 \pm 1.5$ \\
5\% SF-BCP-20 & $0.83 \pm 0.11$ & $-1.78 \pm 0.12$ & $41.3 \pm 3.1$ & $0.61 \pm 0.12$ & $2.6 \pm 1.5$ \\
2\% SF-BCP-20 & $0.82 \pm 0.12$ & $-1.26 \pm 0.09$ & $36.0 \pm 4.2$ & $0.40 \pm 0.09$ & - \\
0\% SF-BCP-20 & $0.44 \pm 0.08$ & $-0.55 \pm 0.05$ & $32.7 \pm 3.8$ & $0.09 \pm 0.05$ & $8.8 \pm 1.5$ \\
\hline
\end{tabular}

